\title{
Update: Multinational listeriosis outbreak due to 'Quargel', a sour milk curd cheese, caused by two different L. monocytogenes serotype 1/2a strains, 2009- 2010
}

R Fretz (rainer.fretz@ages.at) ${ }^{1}$, J Pichler ${ }^{1}$, U Sage ${ }^{1,2}$, P Much $^{1}$, W Ruppitsch ${ }^{1}$, A T Pietzka ${ }^{1}$, A Stöger ${ }^{1}$, S Huhulescu ${ }^{1}$, S Heuberger $^{1}$,

G Appl $^{1}$, D Werber ${ }^{3}$, K Stark 3 , R Prager ${ }^{3}$, A Flieger ${ }^{3}$, R Karpíšková ${ }^{4}$, G Pfaff ${ }^{5}$, F Allerberger ${ }^{1}$

1. Austrian Agency for Health and Food Safety (AGES), Vienna, Austria

2. Binational Consiliar Laboratory for Listeria, Germany and Austria, Vienna, Austria

3. Robert Koch Institute (RKI), Berlin and Wernigerode, Germany

4. National Institute of Public Health, Prague, Czech Republic

5. State Health Office (LGA) Baden-Württemberg, Stuttgart, Germany

Citation style for this article:

Citation style for this article: Fretz R, Pichler J, Sagel U, Much P, Ruppitsch W, Pietzka AT, Stöger A, Huhulescu S, Heuberger S, Appl G, Werber D, Stark K, Prager R, Flieger A, Karpíšková R, Pfaff G, Allerberger F. Update: Multinational listeriosis outbreak due to 'Quargel', a sour milk curd cheese, caused by two different L. monocytogenes serotype 1/2a strains, 2009-2010. Euro Surveill. 2010;15(16):pii=19543. Available online: http://www.eurosurveillance.org/ViewArticle. aspx?Articleld $=19543$

This article has been published on 22 April 2010

We previously reported an outbreak of listeriosis in Austria and Germany due to consumption of 'Quargel' cheese. It comprised 14 cases (including five fatalities) infected by a serotype $1 / 2 a$ Listeria monocytogenes (clone 1), with onset of illness from June 2009 to January 2010. A second strain of $L$. monocytogenes serotype $1 / 2 a$ (clone 2 ) spread by this product could be linked to further 13 cases in Austria (two fatal), six in Germany (one fatal) and one case in the Czech Republic, with onset of disease from December 2009 to end of February 2010.

\section{Clone 1}

As reported earlier, the binational Austrian-German Consiliar Laboratory for Listeria in Vienna noticed a cluster of human isolates of Listeria monocytogenes serotype 1/2a in August 2009 with a new pulsed-field gel electrophoresis (PFGE) pattern [1]. Fourteen cases (12 Austrian and two German), including five with fatal outcome (two of them German), were identified. Onset of disease ranged from June 2009 to January 2010. An epidemiological investigation revealed 'Quargel' cheese produced by an Austrian manufacturer as the source of infection. The product was withdrawn from the Austrian, German, Slovakian and Czech markets on 23 January 2010 [1].

Microbiological investigations confirmed the presence of this new strain (clone 1) in 'Quargel' samples taken at the factory in 2010: Two of 64 isolates available for testing ( 44 isolates cultured from cheese produced in 2010 and provided by the manufacturer, 20 isolates cultured from samples officially gained during outbreak investigation) showed the new PFGE pattern associated with the outbreak.

\section{Clone 2}

The 62 remaining food isolates showed a different PFGE pattern, that had not previously been seen in Austrian isolates either (clone 2). It was indistinguishable from the pattern of a human isolate from a listeriosis patient hospitalised at the time, who claimed to have eaten 'Quargel' cheese. Only two of 46 human L. monocytogenes isolates documented at the Austrian Reference Centre in 2009 yielded this PFGE-pattern, both coming from patients with a food-history positive for 'Quargel'. Ultimately, this second outbreak clone of L. monocytogenes serotype $1 / 2$ a accounted for 13 Austrian cases (two with fatal outcome), six German cases (one death), and one Czech case; onset of disease ranged from December 2009 until end of February 2010. The epidemic curve shows all cases associated with the two different outbreak clones by onset of illness (Figure).

\section{Outbreak analysis}

In total, the outbreak involved 34 cases of invasive listeriosis: 25 outbreak cases originated from seven of nine Austrian provinces. Four of these patients presented with meningitis: two with clone 1 and two with clone 2. A further eight patients were from four of 16 German federal states, and one patient was from the Czech Republic. Eight of the 34 cases in this outbreak had a fatal outcome. The median age of the cases was 72 years (range: 57-89 years), and 26 patients were male. There were no materno-neonatal cases. Underlying diseases were not different from those generally described for patients with listeriosis [2].

A total of 63 food samples of the 'Quargel' cheese products were microbiologically analysed. 20 samples were found positive for L. monocytogenes. 11 of the 20 
samples yielded less than 100 colony-forming units per gram (CFU/g), and nine samples harboured more than $100 \mathrm{CFU} / \mathrm{g}$. All but one case can be explained by consumption of the contaminated product before it was withdrawn from the market on 23 January: one patient who was hospitalised for meningitis on 26 February 2010 had eaten the cheese (purchased before withdrawal from the market) on February 13. A leftover specimen, stored in the patient's refrigerator and sampled on 3 March, yielded 2,100,000 CFU/g of L. monocytogenes.

\section{Case control study}

The source of this outbreak was initially identified based solely on epidemiological findings [1]. We collected the cases' grocery receipts of purchases they made in December 2009, after their discharge from hospital, and compared them for matches. This generated a hypothesis to be tested by a case control study using case-case comparisons. For this study, a case was defined as a person in Austria from whom the L. monocytogenes outbreak clone 1 was isolated. Controls were patients from Austria with

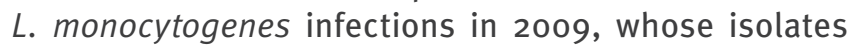
showed other profiles than the outbreak clone 1. Cases were asked about consumption of 12 cheese products in the six-month period prior to disease onset. Control persons were requested to provide information on

\section{FIGURE}

Outbreak cases of listeriosis by onset of illness, Austria, Germany and Czech Republic, 2009-2010 (n=34)

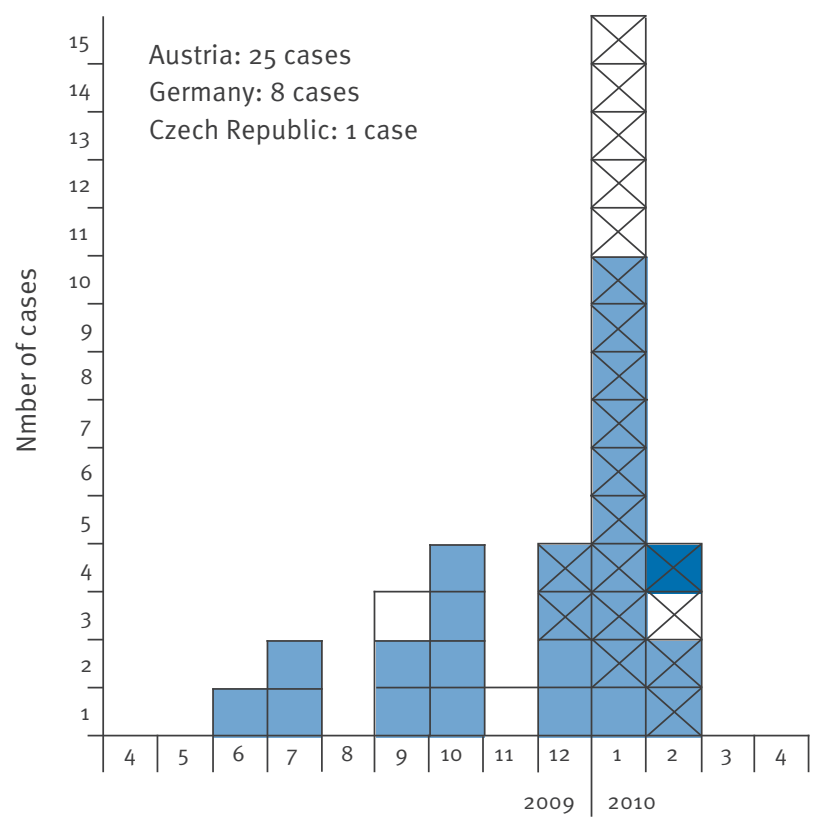

Date (month)

\footnotetext{
$\square$ Austrian cases due to infection with outbreak clone $1(\mathrm{n}=12)$

$\varangle$ Austrian cases due to infection with outbreak clone $2(n=13)$

$\square$ German cases due to infection with outbreak clone $1(n=2)$

$\varangle$ German cases due to infection with outbreak clone $2(n=6)$

Czech case due to infection with outbreak clone $2(n=1)$
}

consumption of the same cheese products in the year 2009. The overall response rate was $83.3 \%$ in the case group (ten of at the time 12 possible cases) and $72.2 \%$ in the control group (24 of at the time 33 possible controls, i.e. listeriosis-patients with isolates that showed other profiles than the outbreak clone 1). Consumption of the 'Quargel' cheese was identified as the only significant risk factor, highly associated with the illness in question. Nine of the ten cases with clone 1 had consumed the product; the tenth case provided no answer concerning this food item. Of 22 control cases (none with clone 2) all but two denied having eaten this specific cheese; the remaining two provided no answer concerning this food item. The computed odds ratio was 76.6 (95\% confidence interval (Cl): 9.3-infinity; P value $<0.001)$.

\section{Conclusions}

The described outbreak provides some valuable lessons: Firstly, it underlines the considerable potential of molecular subtyping as a tool to identify outbreaks. Without routine PFGE typing of human isolates, this outbreak would have been missed. Secondly, it shows impressively that the waning of an outbreak (i.e. disappearance of an outbreak clone) does not necessarily imply that the underlying problem has disappeared. The shift to a different outbreak clone in December 2009/ January 2010 was probably caused by a change (in late November 2009) of the commercial ripening culture used in the cheese factory due to short supply of the original culture. Thirdly, our outbreak also emphasises the considerable potential of cross-border cooperation for elucidating chains of infections in multinational outbreaks. Industrial food production combined with international marketing of food and the low attack rate of $L$. monocytogenes hinder epidemiological outbreak investigations with traditional concepts [2]. Finally, the case of our patient with meningitis who had a leftover specimen of the causative food still in his refrigerator, underlines the importance of visiting households of listeriosis patients in order to obtain food samples and to advise other household members on precautionary measures. A single leftover food sample could prove an invaluable clue for elucidating the source of infection and thereby preventing further illness.

\footnotetext{
References

1. Fretz R, Sagel U, Ruppitsch W, Pietzka AT, Stöger A, Huhulescu $\mathrm{S}$, et al. Listeriosis outbreak caused by acid curd cheese 'Quargel', Austria and Germany 2009. Euro Surveill. 2010;15(5) pii=19477. Available from: http://www.eurosurveillance.org/ ViewArticle.aspx?Articleld $=19477$

2. Allerberger F, Wagner M. Listeriosis: a resurgent foodborne infection. Clin Microbiol Infect. 2010;16(1):16-23.
} 